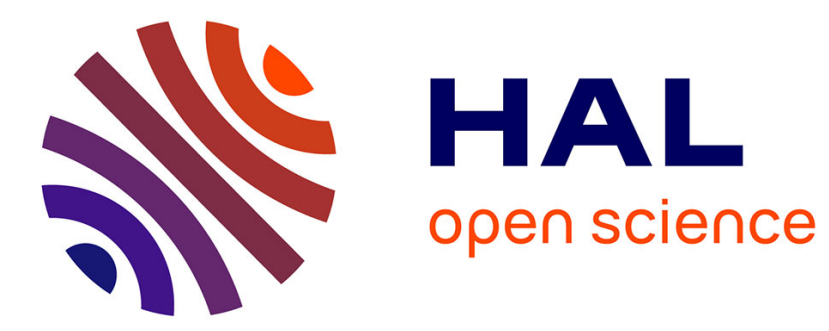

\title{
Nanosecond spin coherence of excitons bound to acceptors in a CdTe quantum well
}

\author{
P. Grinberg, Frédéric Bernardot, Benoît Eble, G. Karczewski, Christophe
}

Testelin, Maria Chamarro

\section{- To cite this version:}

P. Grinberg, Frédéric Bernardot, Benoît Eble, G. Karczewski, Christophe Testelin, et al.. Nanosecond spin coherence of excitons bound to acceptors in a CdTe quantum well. Journal of Applied Physics, 2016, 119 (12), pp.123906. 10.1063/1.4944938 . hal-01310916

\section{HAL Id: hal-01310916 https://hal.sorbonne-universite.fr/hal-01310916}

Submitted on 3 May 2016

HAL is a multi-disciplinary open access archive for the deposit and dissemination of scientific research documents, whether they are published or not. The documents may come from teaching and research institutions in France or abroad, or from public or private research centers.
L'archive ouverte pluridisciplinaire HAL, est destinée au dépôt et à la diffusion de documents scientifiques de niveau recherche, publiés ou non, émanant des établissements d'enseignement et de recherche français ou étrangers, des laboratoires publics ou privés. 


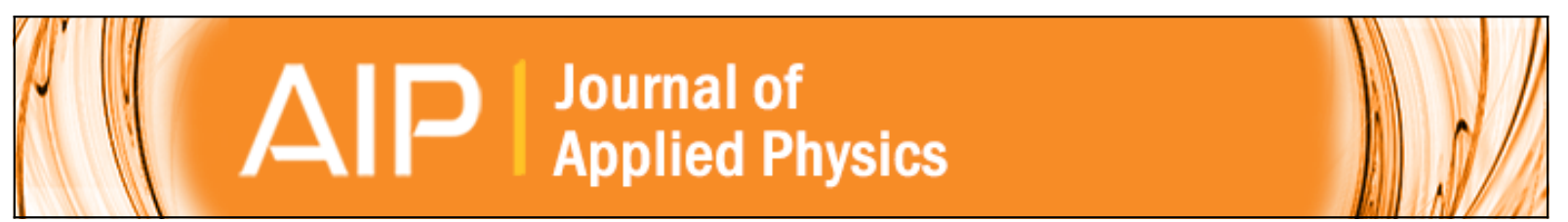

\section{Nanosecond spin coherence of excitons bound to acceptors in a CdTe quantum well}

P. Grinberg, F. Bernardot, B. Eble, G. Karczewski, C. Testelin, and M. Chamarro

Citation: Journal of Applied Physics 119, 123906 (2016); doi: 10.1063/1.4944938

View online: http://dx.doi.org/10.1063/1.4944938

View Table of Contents: http://scitation.aip.org/content/aip/journal/jap/119/12?ver=pdfcov

Published by the AIP Publishing

\section{Articles you may be interested in}

Time-resolved photoluminescence spectroscopy of localized exciton magnetic polarons in Cd0.70Mn0.30Te spin glass compound

J. Appl. Phys. 115, 133709 (2014); 10.1063/1.4870645

Long-lived, room-temperature electron spin coherence in colloidal CdS quantum dots

Appl. Phys. Lett. 100, 122406 (2012); 10.1063/1.3696069

Transient spectral dependence of photoinduced magneto-optical Faraday effect in CdTe quantum dots AIP Advances 2, 012116 (2012); 10.1063/1.3679403

Electron spin coherence in $\mathrm{n}$-doped $\mathrm{Cd} \mathrm{Te} / \mathrm{Cd} \mathrm{Mg}$ Te quantum wells

Appl. Phys. Lett. 89, 221113 (2006); 10.1063/1.2397552

Spatial diffusion of excitons in $\mathrm{n}$-type modulation-doped $(\mathrm{Cd}, \mathrm{Mn}) \mathrm{Te} /(\mathrm{Cd}, \mathrm{Mg}) \mathrm{Te}$ single quantum wells under magnetic fields

Appl. Phys. Lett. 83, 2853 (2003); 10.1063/1.1614838

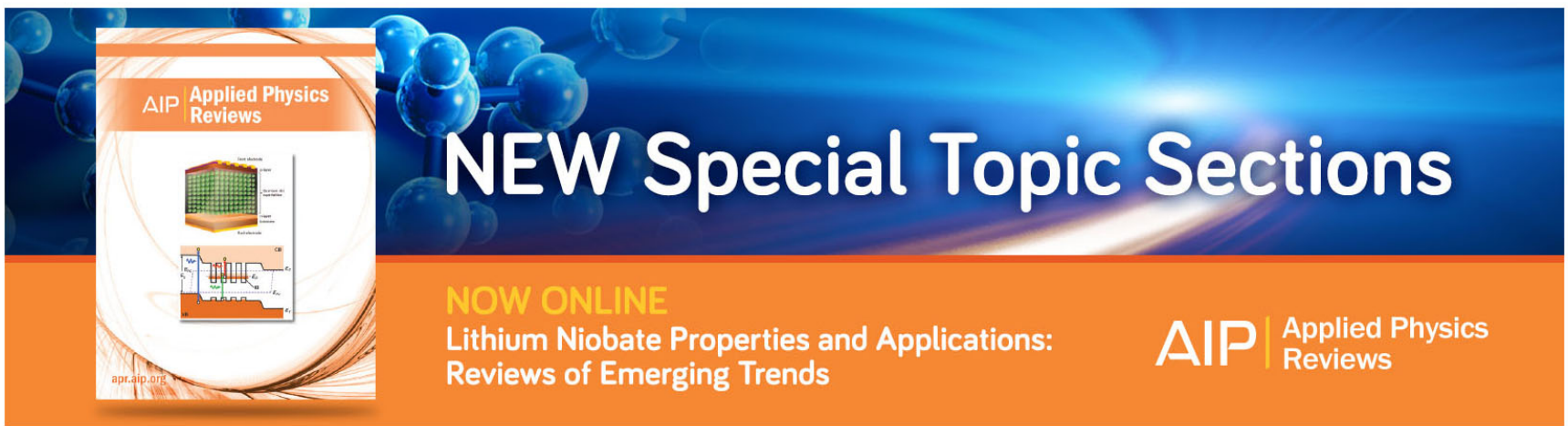




\title{
Nanosecond spin coherence of excitons bound to acceptors in a CdTe quantum well
}

\author{
P. Grinberg, ${ }^{1}$ F. Bernardot, ${ }^{1,2}$ B. Eble,${ }^{1}$ G. Karczewski, ${ }^{3}$ C. Testelin, ${ }^{1}$ and M. Chamarro ${ }^{1}$ \\ ${ }^{1}$ Sorbonne Universités, UPMC Univ Paris 06, CNRS-UMR 7588, Institut des NanoSciences de Paris, F-75005 \\ Paris, France \\ ${ }^{2}$ Univ Paris 07, F-75205 Paris Cedex 13, France \\ ${ }^{3}$ Polish Acad Sci, Inst Phys, PL-02668 Warsaw, Poland
}

(Received 7 October 2015; accepted 16 March 2016; published online 31 March 2016)

\begin{abstract}
We have studied the coherent spin dynamics of excitons bound to acceptors, $\mathrm{A}^{0} \mathrm{X}$, immersed in a CdTe quantum well by using time resolved photo-induced Faraday rotation. We have also measured the time-resolved differential transmission in order to determine a $\mathrm{A}^{0} \mathrm{X}$ lifetime of $220 \mathrm{ps}$, which is independent of the applied magnetic field. We show that at low magnetic field, the spin of $\mathrm{A}^{0} \mathrm{X}$ is completely frozen during a time, $\cong 4.5 \mathrm{~ns}$, at least twenty times longer than its lifetime. We compare the spin properties of $\mathrm{A}^{0} \mathrm{X}$ with the spin properties of other charged excitons systems, and we conclude that the hyperfine interaction of the photo-created electron spin with nuclear spins is very likely to be at the origin of the observed spin dephasing times. C 2016 AIP Publishing LLC.

[http://dx.doi.org/10.1063/1.4944938]
\end{abstract}

\section{INTRODUCTION}

The introduction of dopants in semiconductors allows the control of electric, optical, and magnetic properties of devices. Owing to the continuous downscaling of semiconductor devices, single dopant atoms are now becoming increasingly important and researched, not only for classical electronics but also in the field of quantum information. ${ }^{1-3}$

As with trapped ions in cold gases, paramagnetic impurities trapped on a semiconductor lattice have uniform properties and relatively long spin lifetimes. ${ }^{4,5}$ In particular, single excitons, electrons, or holes trapped by individual donors or acceptors in semiconductors at low temperatures are promising spinqubit candidates due to the combination of the advantages of semiconductor and atomic systems. Their semiconductor environment provides a natural localization and ease of fabrication that is absent in atomic and ion qubit systems. Scalability is a common property with other solid-state systems as semiconductor quantum dots (QDs) or nitrogen vacancy centers, but the former ones are rarely identical and the emission efficiency of the latter ones in the zero-phonon line is small $(3 \%){ }^{6}$

In this paper, we focus our study on the coherent spin evolution of excitons bound to acceptors immersed in a CdTe QW. This system increases the localization of the electron wave function, with respect to its localization on donors or acceptors in three-dimensional crystals, and is also known to purify the optical selection rules for circularly polarized light of excitons bound to the donors, $\mathrm{D}^{0} \mathrm{X},{ }^{7}$ and then allows a higher degree of optical orientation of the electron spins than in 3D crystals. Moreover, hyperfine interaction of localized electrons with nuclear moments of CdTe atoms is weaker than for III-V compounds ${ }^{8}$ and can be reduced or suppressed by isotopic purification.

\section{SAMPLE AND MEASUREMENTS}

The studied sample consists of a CdTe/CdMgTe heterostructure grown by molecular-beam epitaxy on a (100)-oriented
GaAs substrate and containing a $80 \AA \mathrm{CdTe}$ QW. It is nominally undoped, but due to residual impurities (I, N), the QW contains donors and acceptors in very small concentrations. We used a degenerate pump-probe technique, the Photoinduced Faraday Rotation (PFR), which is well-adapted to study spin relaxation and decoherence times of both excited states and resident electrons or holes. Laser pulses with a 2 ps duration were generated by a Ti:Sapphire mode-locked oscillator at $75.8 \mathrm{MHz}$. The pump beam polarization is $\sigma+/ \sigma-$ modulated at $400 \mathrm{kHz}$ with an electro-optic modulator (average intensity $\approx 1 \mathrm{~W} / \mathrm{cm}^{2}$ ); the probe beam is linearly polarized, and its intensity $\left(\approx 0.1 \mathrm{~W} / \mathrm{cm}^{2}\right)$ is modulated with an acousto-optic modulator at $3 \mathrm{kHz}$. We have chemically suppressed the GaAs substrate in order to measure, after transmission through the sample, the rotation angle of the probe beam polarization with an optical bridge. The sample was placed in a liquid-helium cryostat and a magnetic field was applied perpendicularly to the growth direction of the sample, in Voigt geometry, using a superconducting splitcoil magnet.

\section{RESULTS AND DISCUSSION}

The $2 \mathrm{~K}$-photoluminescence (PL) spectrum of the sample, excited at $1.96 \mathrm{eV}$, is shown in Fig. 1(a). It is dominated by the recombination of donor-bound excitons $(1.6236 \mathrm{eV}$, $\left.\mathrm{D}^{0} \mathrm{X}\right)$, shows a low-energy peak related to the recombination of acceptor-bound excitons $\left(1.6207 \mathrm{eV}, \mathrm{A}^{0} \mathrm{X}\right)$, and a higher energy shoulder related to the free exciton emission (1.6262 eV, X). Fig. 1(b) shows the PFR signal obtained at $2 \mathrm{~K}$ in magnetic field, $522.5 \mathrm{mT}$, and for different energies of the pump and probe beams. The PFR signal shows longliving oscillations for energies ranging from $1.626 \mathrm{eV}$ to $1.614 \mathrm{eV}$, and their amplitude becomes smaller for the lowest energies, between $1.622 \mathrm{eV}$ and $1.614 \mathrm{eV}$. This oscillatory signal has been associated in previous works ${ }^{7}$ to the coherent precession of the oriented electron bound to a neutral donor 

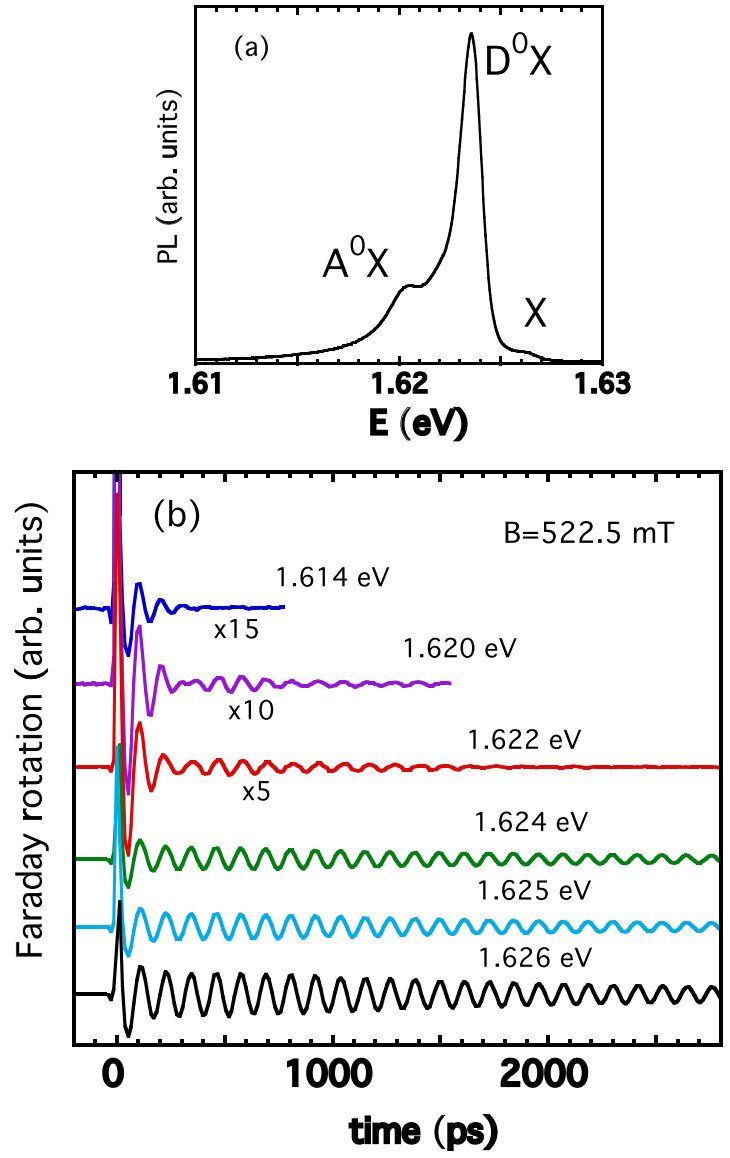

FIG. 1. (a) Inset: $2 \mathrm{~K}-\mathrm{PL}$ spectrum of the $80 \AA \mathrm{CdTe} \mathrm{QW}$, nominally undoped. (b) $2 \mathrm{~K}$-PFR signal versus pump-probe delay time, obtained for a fixed value of the transverse magnetic field and for different energies. As in the other figures of this article, the PFR curves are vertically shifted for clarity.

$\mathrm{D}^{0}$. The pump pulse creates $\mathrm{X}$ or $\mathrm{D}^{0} \mathrm{X}$; after capture of free excitons on donors and the subsequent flip of the hole spin of $\mathrm{D}^{0} \mathrm{X}$ and its recombination, we recover a spin polarized $\mathrm{D}^{0}$. At low energy (1.621-1.614 eV), we have also observed in PFR curves a short-living oscillatory component in the range of several hundreds of ps. This fast component, combined with the long-living one, gives rise to beating patterns in the PFR signal (see Figure 2(a)). At $1.614 \mathrm{eV}$ (see Fig. 1(b)), only the short-living oscillatory signal is present. As discussed later, this short-living oscillatory signal is associated with the coherent spin dynamics of acceptor-bound excitons, $\mathrm{A}^{0} \mathrm{X}$.

Figure 2(b) shows the PFR signal obtained at $1045 \mathrm{mT}$ near the maximum of PL $(1.624 \mathrm{eV})$. Because the absorption of the $\mathrm{X}$, at this energy, is at least ten times more important than the absorption of the $\mathrm{D}^{0} \mathrm{X}$, the pump beam creates free excitons in the tail of $\mathrm{X}$ absorption band. We have, then, fitted the curve with three components, which are associated with (i) the spin polarization of $\mathrm{D}^{0}$, (ii) the $\mathrm{X}$ contribution, and (iii) the $\mathrm{D}^{0} \mathrm{X}$ contribution. We associated two damped cosines to the first two contributions. The precession frequencies are the Larmor frequencies of $\mathrm{X}$ and $\mathrm{D}^{0}, \Omega_{X, e}$ $=\frac{g_{\perp}^{X, e} \mu_{B}}{\hbar} B$. By fitting the PFR signal obtained at different magnetic fields to a three-component expression, as in Figure 2(b), we are able to obtain $\Omega_{X, e}$ as a function of the

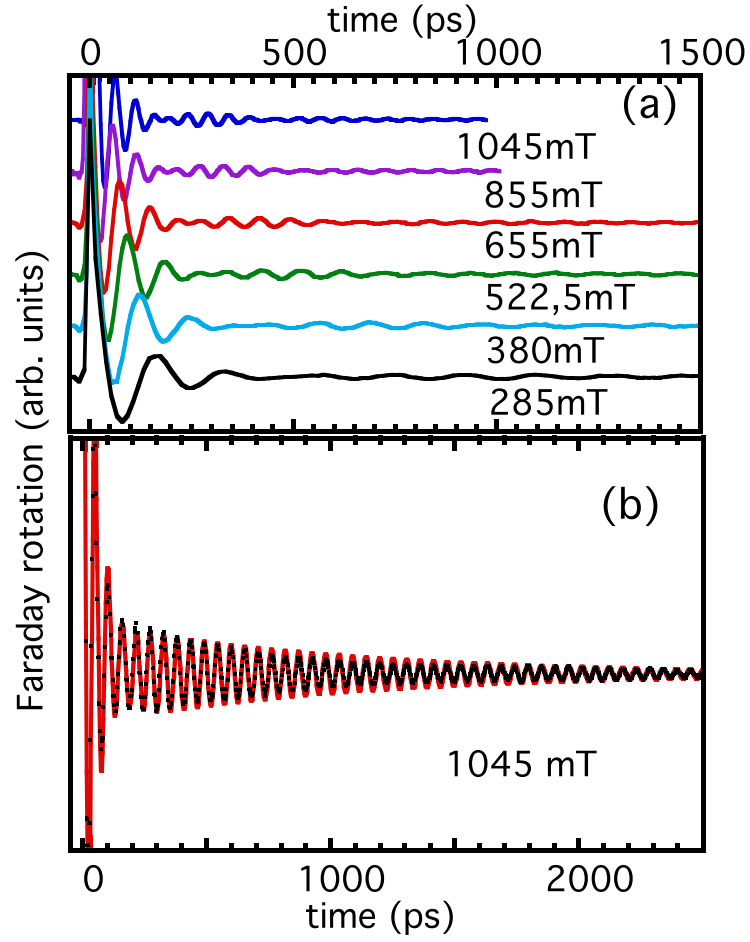

FIG. 2. (a) $2 \mathrm{~K}-\mathrm{PFR}$ signal versus time obtained at $1.621 \mathrm{eV}$ for different magnetic fields. (b) $2 \mathrm{~K}$-PFR signal versus time obtained at $1.624 \mathrm{eV}$ for $\mathrm{B}=1045 \mathrm{mT}$. Dots represent experimental data and red solid line represents the fit to the expression $0.009 \exp (-t / 1000) \cos (0.1155 t)+0.075 \exp$ $(-t / 50) \cos (0.128 t)+0.02 \exp (-t / 60)$.

applied magnetic field, and then to determine the transverse Landé factors for the free exciton $\left(g_{\perp}^{X}\right)$ and for the bound electron $\left(g_{\perp}^{e}\right)$. We obtain $\left|g_{\perp}^{X}\right|=1.42$, which is close to the value found in a CdTe $80 \AA$ QW by Sirenko et al. ${ }^{9}$ and $\left|g_{\perp}^{e}\right|=1.23$ (see Figure 3(a)), which is also a value similar to the one obtained previously in a I-doped CdTe QW. ${ }^{7}$

The $\mathrm{D}^{0} \mathrm{X}$ contains three particles, two electrons with antiparallel spins and one hole. The spin of $\mathrm{D}^{0} \mathrm{X}$ is then fixed by the hole spin. For holes in QWs $\left|g_{\perp}^{h}\right| \approx 0,{ }^{9}$ as a consequence, the associated Larmor frequency is almost zero, and the contribution of the excited state $\mathrm{D}^{0} \mathrm{X}$ is a single exponential. Hole spin relaxation time, $T_{s}^{h}$, in $\mathrm{D}^{0} \mathrm{X}$ can be obtained using $1 / T^{D^{0} X}=1 / T_{\text {lifetime }}^{D^{0} X}+1 / T_{s}^{h}$, which reflects the fact that there are two possible channels through which the difference of spin populations of $\mathrm{D}^{0} \mathrm{X}$ decreases: the recombination of $\mathrm{D}^{0} \mathrm{X}$ and the hole spin flip. We have measured a lifetime of $\mathrm{D}^{0} \mathrm{X}$ equal to $T_{\text {lifetime }}^{D^{0} X}=200 \mathrm{ps}$ from timeresolved PL detected at $1.6236 \mathrm{eV}$ and excited at $2.147 \mathrm{eV}$ in $\mathrm{B}=0 \mathrm{~T}$. From the value $T^{D^{0} X}=60 \mathrm{ps}$ extracted from the fit of Fig. 2(b) and assuming that the lifetime does not change with the magnetic field, we obtain a spin relaxation time $T_{s}^{h}=83 \mathrm{ps}$ at about $1 \mathrm{~T}$, which compares well with the hole dephasing time found at $1 \mathrm{~T}$ by Zhukov et al. in a $20-\mathrm{nm}$ thick CdTe QW containing a two-dimensional (2D) hole gas at low concentration (less than $10^{10} \mathrm{~cm}^{-2}$ ). ${ }^{10} T_{s}^{h}=83 \mathrm{ps}$ is also in good agreement with our previous results in a I-doped $\mathrm{QW},{ }^{7}$ but is longer than the hole spin relaxation time obtained at zero magnetic field for a $77 \AA \mathrm{CdTe}$ QW containing a 2D hole gas with concentration $7 \times 10^{10} \mathrm{~cm}^{-2}$. ${ }^{11}$ Spin relaxation 
B (T)

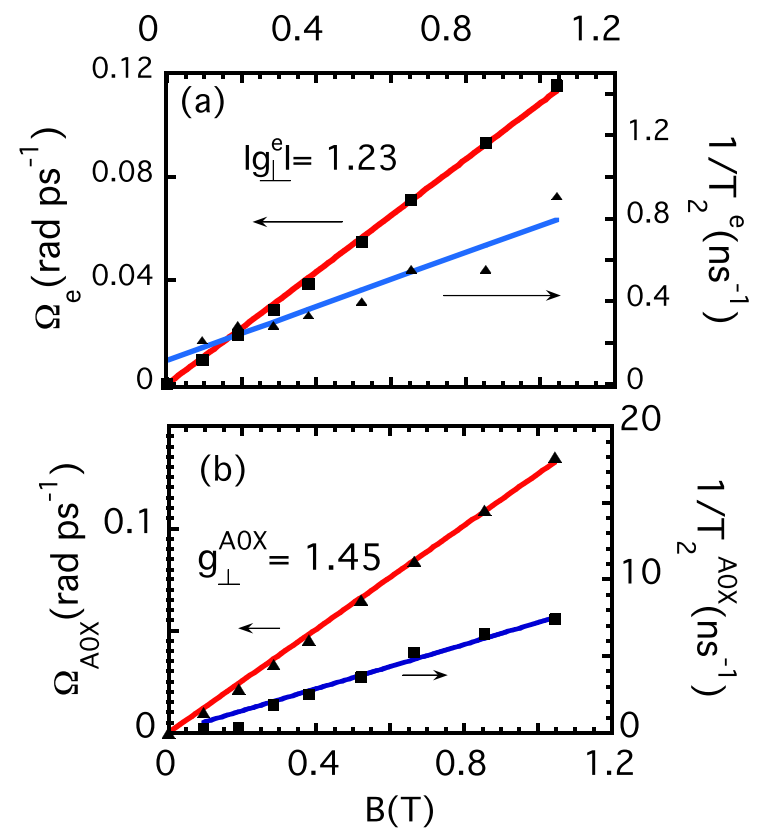

FIG. 3. (a) Experimental data of the $\mathrm{D}^{0}$ Larmor frequency (squares) and the $\mathrm{D}^{0}$ dephasing rate (triangles) as a function of the applied magnetic field. Red and blue solid lines are linear fits described in the text. (b) Experimental data of the $\mathrm{A}^{0} \mathrm{X}$ Larmor frequency (squares) and the $\mathrm{A}^{0} \mathrm{X}$ dephasing rate (triangles) as a function of the applied magnetic field. Red and blue solid lines are linear fits described in the text.

processes are significantly suppressed for localized holes or electrons. At low temperature, when the concentration of a hole spin gas is low, holes are localized by QW potential fluctuations associated with spatial variations of the density of acceptors in the barrier, and this fact could explain the difference with the spin relaxation times measured in the mentioned samples containing a $2 \mathrm{D}$ hole gas. $\mathrm{D}^{0} \mathrm{X}$ in our sample shows a binding energy $\approx 2 \mathrm{meV}$, see Fig. 1(a).

Figure 4 shows the PFR signal obtained at $1.614 \mathrm{eV}$ as a function of the magnetic field. At $\mathrm{B}=0 \mathrm{~T}$, the PFR signal has a two-exponential behaviour with a very fast component (15 ps) and a slower one (220 ps). At $1.614 \mathrm{eV}$, we excite

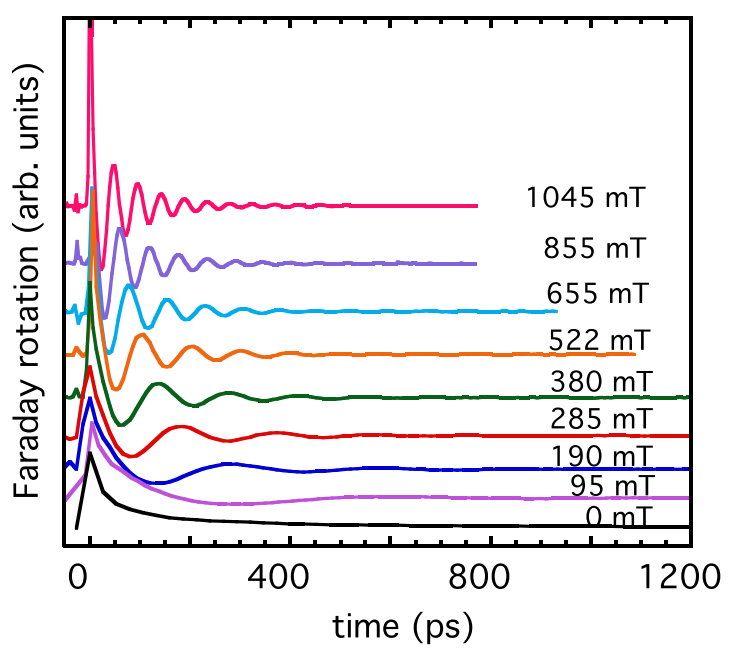

FIG. 4. 2K-PFR signal at $1.614 \mathrm{eV}$ as a function of time and for different transverse magnetic fields. mainly the $\mathrm{A}^{0} \mathrm{X}$ state in its low-energy tail. To determine the lifetime of this state, we have performed differential transmission measurements, because the luminescence is too weak to be measured in a time-resolved PL experiment. The differential transmission curve obtained at zero magnetic field is given in Fig. 5(a). An offset has been added to the experimental curve. We underline that this curve does not change significantly when a magnetic field is applied until $1045 \mathrm{mT}$. The amplitude of this signal is weaker than the PFR one, but the fit is very similar and gives two exponential decays with similar characteristic times; in particular, we determine a lifetime of $\mathrm{A}^{0} \mathrm{X}, T_{\text {lifetime }}^{A^{0} X}=220 \mathrm{ps}$. The $\mathrm{A}^{0} \mathrm{X}$ contains three particles, two holes with antiparallel spins and one electron. The spin of $\mathrm{A}^{0} \mathrm{X}$ is then fixed by the photocreated electron spin. The spin dephasing time $T_{2}^{A^{0} X}$ of the $\mathrm{A}^{0} \mathrm{X}$ can be obtained through $1 / T^{A^{0} X}-1 / T_{\text {lifetime }}^{A^{0} X}=1 / T_{2}^{A^{0} X}$, where $\mathrm{T}^{\mathrm{A} 0 \mathrm{X}}$ is extracted from the fit of the PFR curve. At zero magnetic field, we obtain $T_{\text {lifetime }}^{A^{0} X} \approx T^{A^{0} X} \ll T_{2}^{A^{0} X}$. We have fitted the PFR curves at $\mathrm{B} \neq 0$ to a damped cosine oscillating at the $\mathrm{A}^{0} \mathrm{X}$ Larmor frequency, $\Omega_{A^{0} x}$, and obtained a transverse Landé factor $\left|g_{\perp}^{A^{0} X}\right|=1.45$ (Figure 3(b)). Figure 5(b) shows the PFR signal and its corresponding fit, at $1.614 \mathrm{eV}$ and for $\mathrm{B}=1045 \mathrm{mT}$. For $95 \mathrm{mT}$, we determine $T_{2}^{A^{0} X} \approx 4.5 \mathrm{~ns}$ (see Fig. 3(b)). This value is almost two orders of magnitude larger than the one reported by Vanelle et al. ${ }^{11}$ for the relaxation time of the electron spin in a positively charged exciton in CdTe QWs, $60 \mathrm{ps}$, but is comparable to

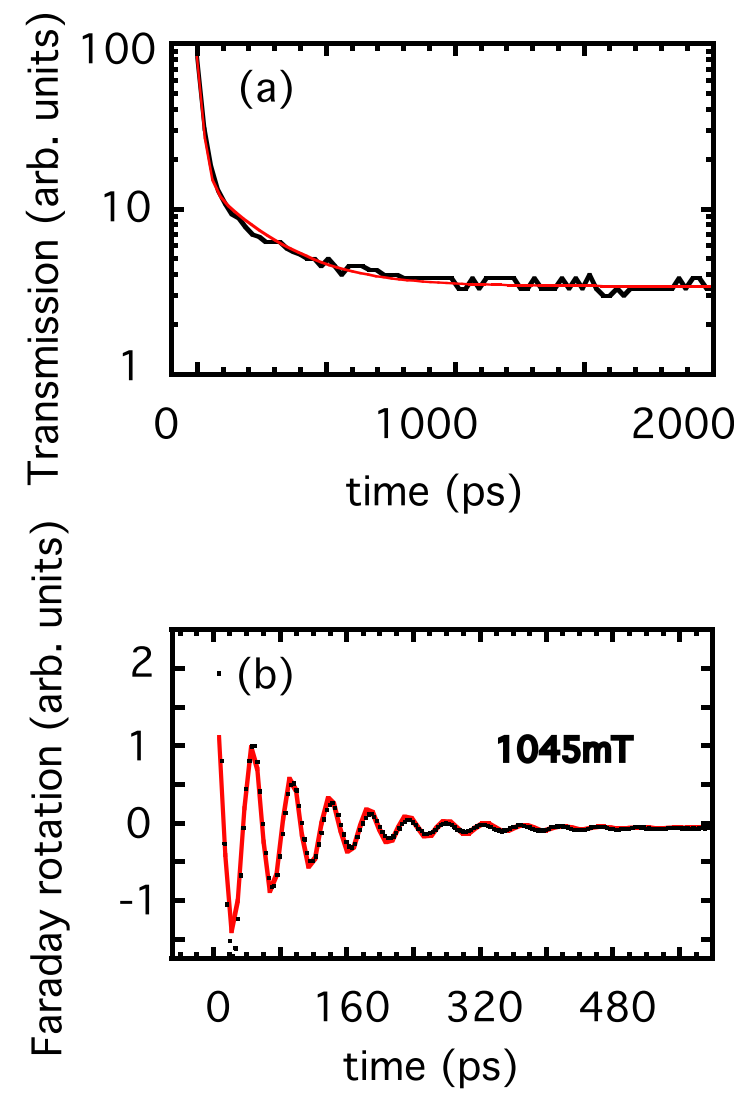

FIG. 5. (a) Time-resolved differential transmission obtained at $\mathrm{B}=0,2 \mathrm{~K}$ and $1.614 \mathrm{eV}$. (b) Squares represent the PFR signal obtained at $1.614 \mathrm{eV}$ and $\mathrm{B}=1045 \mathrm{mT}$. The red solid line is a theoretical fit. 
the one measured for resident electrons in $\mathrm{n}$-doped CdTe QDs at $250 \mathrm{mT}$ (Ref. 12) and with the value obtained for 2D electron gas in a CdTe QW at low concentration ${ }^{13}$ or for $\mathrm{D}^{0}$ in this work and in a previous work. ${ }^{7}$

$T_{2}^{A^{0} X}$ decreases as the magnetic field increases, and the dephasing rate $1 / T_{2}^{A^{0} X}$ shows a linear dependence on $\mathrm{B}$ which evidences that the dephasing is dominated by the inhomogeneities of $g_{\perp}^{A^{0} X}$. A linear fit of $1 / T_{2}^{A 0 X}$ versus applied magnetic field (Fig. 3(b)) to

$$
\frac{1}{T_{2}^{A^{0} X}}(B)=\frac{1}{T_{2,0}^{A^{0} X}}+\Delta g_{\perp}^{A^{0} X} \frac{\mu_{B}}{\hbar} B
$$

gives $\frac{\Delta g_{\perp}^{A^{0} X}}{\mid g_{\perp}^{A^{0} \mid}} \approx 5 \% ; \frac{1}{T_{20}^{A^{0} X}}$ is the $\mathrm{A}^{0} \mathrm{X}$ spin dephasing rate at $\mathrm{B}=0 \mathrm{~T}$, and $\Delta g_{\perp}^{A^{0} X}$ is the dispersion on the $\mathrm{A}^{0} \mathrm{X}$ Landé factor. We have also observed a linear dependence of the $\mathrm{D}^{0}$ dephasing rate, $1 / T_{2}^{e}$, versus $\mathrm{B}$, and have deduced from the fit shown in the inset of Fig. 3(a) a much smaller value of $\frac{\Delta g_{\perp}^{e}}{\left|g_{\perp}^{e}\right|} \approx 0.6 \%$ and $T_{2}^{e}(B=0) \cong 9 \mathrm{~ns}$. The latter ratios indicate that the inhomogeneities of $g_{\perp}^{A^{0} X}$ are larger than the ones of $g_{\perp}^{e}$, and are similar to the $\frac{\Delta g_{\perp}^{e}}{\left|g_{\perp}^{e}\right|}$ values obtained in n-doped ${ }^{12,14}$ or p-doped QDs. ${ }^{15}$

At $\mathrm{B}=0 \mathrm{~T}$, at very small concentration, and because of the localization of the donor-bound electron spins, their dephasing time is likely to be determined by hyperfine interaction. ${ }^{16,17}$ The studied $\mathrm{A}^{0} \mathrm{X}$ shows a binding energy of $\approx$ $12 \mathrm{meV}$. Moreover, as for positively charged excitons in QDs, ${ }^{18}$ the exchange interaction between the electron and the two holes cancels in the $\mathrm{A}^{0} \mathrm{X}$ fundamental state. Then, at low concentration of acceptors, ${ }^{19}$ we expect that the spin dephasing time of the electron spin in $\mathrm{A}^{0} \mathrm{X}$ should also be determined by hyperfine interaction. If $\mathrm{A}_{\mathrm{i}}$ is the hyperfine constant for the non-zero nuclear spin $I_{i}$ with abundance $p_{i}$ in the crystal lattice, the dephasing time due to the hyperfine interaction writes

$$
T_{\Delta}=\hbar \frac{\sqrt{3 N_{L}}}{\sqrt{2 n \sum_{i} I_{i}\left(I_{i}+1\right)\left(A_{i}\right)^{2} p_{i}}},
$$

where $\mathrm{n}=2$ is the number of nuclei inside a unit cell. The different isotopes of $\mathrm{Cd}$ and Te have the same $\mathrm{A}_{\mathrm{i}}: \mathrm{A}_{\mathrm{Cd}}=31 \mu \mathrm{eV}$ and $\mathrm{A}_{\mathrm{Te}}=45 \mu \mathrm{eV} . \quad \mathrm{I}^{\mathrm{Cd}}=\mathrm{I}^{\mathrm{Te}}=1 / 2 \quad$ with $\quad \mathrm{p}_{\mathrm{Cd}}=0.25$ and $\mathrm{p}_{\mathrm{Te}}=0.08$.

We estimate the localization radius of $\mathrm{A}^{0} \mathrm{X}$ to be $4.5 \mathrm{~nm}$; then we obtain $\mathrm{N}_{\mathrm{L}}=3.5 \times 10^{4}$ and, finally, calculate $T_{\Delta}=6.1 \mathrm{~ns}$ which is in good agreement with our results.

Contrary to the p-doped InAs QDs for which the optical initialization of the hole spin has been demonstrated at $\mathrm{B}=0$ by the creation of positively charged excitons, ${ }^{20,21}$ the resonant excitation of $\mathrm{A}^{0} \mathrm{X}$ in CdTe QWs does not allow the optical initialization of an acceptor-bound hole spin, $\mathrm{A}^{0}$. The reason is the very strong quenching of spin dephasing during the lifetime of $\mathrm{A}^{0} \mathrm{X}$. However, in a transverse magnetic field, the $\mathrm{A}^{0} \mathrm{X}$ recombination should lead to hole spin polarization along the propagation direction of light when the condition $\Omega_{A^{0} X} T_{\text {lifetime }}^{A^{0} X} \gg 1$ is fulfilled, ${ }^{22,23}$ as in our case for B $>0.6 \mathrm{~T}$. In PFR curves of
Figure 4, it is difficult to obtain a clear contribution of the oriented hole spin, i.e., an extra exponential (if $\left|g_{\perp}^{h}\right|=0$ ) or damped cosine term for the fitted curves. That means at $\mathrm{B}>0.6 \mathrm{~T}$, the hole spin dephasing is very fast $(<10 \mathrm{ps})$ despite an important localization (the binding energy for $\mathrm{N}$ acceptors in bulk is equal to $54.8 \mathrm{meV}$ ). ${ }^{24,25}$ That is in contrast with our results in this work concerning hole spin relaxation in $\mathrm{D}^{0} \mathrm{X}$ and also with spin dephasing times observed for 2D hole gas localized by QW potential fluctuations. ${ }^{10}$ Indeed, the spin state of $\mathrm{A}^{0}$ is strongly dependent on the symmetry of the confinement potential. The Coulomb potential of an acceptor retains the 4-fold degeneracy of hole spin in valence band as long as the impurity potential respects the cubic symmetry of the crystal; ${ }^{26}$ in contrast, the confinement of QDs lifts degeneracy and makes the heavy holes the lower lying states. ${ }^{27}$ More studies are needed to clarify hole spin relaxation mechanisms of $\mathrm{A}^{0}$ when the acceptor atom is immersed in a CdTe QW.

\section{CONCLUSIONS}

In conclusion, we have studied, in the same CdTe QW, the spin dynamics of free excitons, and two kinds of bound excitons, $\mathrm{D}^{0} \mathrm{X}$ and $\mathrm{A}^{0} \mathrm{X}$, as well as the spin dynamics of resident states donor-bound electron, $\mathrm{D}^{0}$, or acceptor-bound hole and $\mathrm{A}^{0}$. We have determined the respective transverse Landé factors and dephasing times. In particular, at low magnetic field, we have shown that localization of $\mathrm{A}^{0} \mathrm{X}$ enhances by almost two orders of magnitude the spin dephasing time of photo-created electrons leading to a value close to the value obtained for the $\mathrm{D}^{0}$ and exceeding by at least one order of magnitude its lifetime. This spin dephasing quenching during the lifetime of $\mathrm{A}^{0} \mathrm{X}$ avoids the spin polarization of $\mathrm{A}^{0}$. We have not obtained experimental signature of $\mathrm{A}^{0}$ spin polarization either at $0.6<\mathrm{B}<1.2 \mathrm{~T}$, and we have then concluded that the spin state of $\mathrm{A}^{0}$ is more affected by light-heavy mixing character or inhomogeneities of the transverse Landé factors than the hole spin confined by QW potential fluctuations.

\section{ACKNOWLEDGMENTS}

The authors would like to thank Mathieu Bernard for cryogenic help in our experiments. The research in Poland was partially supported by the National Science Center (Grant No. DEC-2014/14/M/ST3/00484) and the Foundation for Polish Science (Master program).

${ }^{1}$ P. M. Koenraad and M. E. Flatté, Nat. Mater. 10, 91 (2011).

${ }^{2}$ G. Ethier-Majcher, P. St-Jean, G. Boso, A. Tosi, J. F. Klem, and S. Francoeur, Nat. Commun. 5, 3980 (2014).

${ }^{3}$ J. van der Heiden, J. Salfi, J. A. Mol, J. Verduijn, G. C. Tettamanzi, A. R. Hamilton, N. Collaert, and S. Rogge, Nano Lett. 14, 1492 (2014).

${ }^{4}$ J. Tisler, G. Balasubramanian, B. Naydenov, R. Kolesov, B. Grotz, R. Reuter, J. P. Boudou, P. A. Curmi, M. Sennour, A. Thorel, M. Borsch, K. Aulenbacher, R. Erdman, P. R. Hemmer, F. Jelezko, and J. Wrachturp, ACS Nano 3, 1959 (2009).

${ }^{5}$ A. M. Tyryshkin, S. Tojo, J. L. Morton John, H. Riemann, N. V. Abrosimov, P. Becker, H. J. Pohl, T. Schenkel, M. L. W. Thewalt, K. M. Itoh, and S. A. Lyon, Nat. Mater. 11, 143 (2012).

${ }^{6}$ A. Beveratos, Thèse Paris XI, 2002. 
${ }^{7}$ J. Tribollet, E. Aubry, G. Karczewski, B. Sermage, F. Bernardot, C. Testelin, and M. Chamarro, Phys. Rev. B 75, 205304 (2007).

${ }^{8}$ C. Testelin, B. Eble, F. Bernardot, G. Karczewski, and M. Chamarro, Phys. Rev. B 77, 235306 (2008).

${ }^{9}$ A. A. Sirenko, T. Ruf, M. Cardona, D. R. Yakolev, W. Ossau, A. Waag, and G. Landwehr, Phys. Rev. B 56, 2114 (1997).

${ }^{10}$ E. A. Zhukov, D. R. Yakovlev, M. Gerbracht, G. V. Mikhailov, G. Karczewski, T. Wojtowicz, J. Kossut, and M. Bayer, Phys. Rev. B 79, 155318 (2009).

${ }^{11}$ E. Vanelle, M. Paillard, X. Marie, T. Amand, P. Gilliot, D. Brinkmann, R. Levy, J. Cibert, and S. Tatarenko, Phys. Rev. B 62, 2696 (2000).

${ }^{12}$ M. Syperek, D. R. Yakovlev, I. A. Yugova, J. Misiewicz, I. V. Sedova, S. V. Sorokin, A. A. Toropov, S. V. Ivanov, and M. Bayer, Phys. Rev. B 84, 085304 (2011).

${ }^{13}$ R. Bratschisch, Z. Chen, S. T. Cundiff, E. A. Zhukov, D. R. Yakovlev, M. Bayer, G. Karczewski, T. Wojtowicz, and J. Kossut, Appl. Phys. Lett. 89, 221113 (2006).

${ }^{14}$ A. Schwan, B. M. Meiners, A. B. Henriques, A. D. B. Maia, A. A. Quivy, S. Spatzek, S. Varwig, D. R. Yakovlev, and M. Bayer, Appl. Phys. Lett. 98, 233102 (2011).

${ }^{15}$ F. Fras, B. Eble, B. Siarry, F. Bernardot, A. Miard, A. Lemaitre, C. Testelin, and M. Chamarro, Phys. Rev. B 86, 161303(R) (2012).

${ }^{16}$ R. I. Dzhioev, K. V. Kavokin, V. L. Korenev, M. V. Lazarev, B. Y. Meltser, M. N. Stepanova, B. P. Zakharchenya, D. Gammon, and D. S. Katzer, Phys. Rev. B 66, 245204 (2002).
${ }^{17}$ I. A. Merkulov, A. M. Efros, and M. Rosen, Phys. Rev. B 65, 205309 (2002).

${ }^{18}$ P. F. Braun, X. Marie, L. Lombez, B. Urbaszek, T. Amand, P. Renucci, V. K. Kalevich, K. V. Kavokin, O. Krebs, P. Voisin, and Y. Masumoto, Phys. Rev. Lett. 94, 116601 (2005).

${ }^{19}$ H. Mathieu, J. Camassel, and F. Ben Chekroun, Phys. Rev. B 29, 3438 (1984).

${ }^{20}$ B. D. Gerardot, D. Brunner, P. A. Dalgarno, P. Ohberg, S. Seidl, M. Kroner, K. Karrai, N. G. Stoltz, P. M. Petroff, and R. J. Warburton, Nature 451, 441 (2008).

${ }^{21}$ B. Eble, P. Desfonds, F. Fras, F. Bernardot, C. Testelin, M. Chamarro, A. Miard, and A. Lemaitre, Phys. Rev. B 81, 045322 (2010); F. Fras, B. Eble, P. Desfonds, F. Bernardot, C. Testelin, M. Chamarro, A. Miard, and A. Lemaitre, ibid. 84, 125431 (2011).

${ }^{22}$ M. V. G. Dutt, J. Cheng, B. Li, X. D. Xu, X. Q. Li, P. R. Berman, D. G. Steel, A. S. Bracker, D. Gammon, S. E. Economou, R. B. Liu, and L. J. Sham, Phys. Rev. Lett. 94, 227403 (2005).

${ }^{23}$ I. A. Yugova, A. A. Sokolova, D. R. Yakovlev, A. Greilich, D. Reuter, A. D. Wieck, and M. Bayer, Phys. Rev. Lett. 102, 167402 (2009).

${ }^{24}$ A. Boudoukha, R. Legros, L. Svob, and Y. Marfaing, J. Cryst. Growth 72, 226 (1985).

${ }^{25}$ M. L. S. Moldavan, P. A. J. Hirsch, C. D. Stinespring, T. H. Myers, and N. C. Giles, J. Electron. Mater. 27, 756 (1998).

${ }^{26}$ A. Baldereschi and N. O. Lipari, Phys. Rev. B 8, 2697 (1973).

${ }^{27}$ D. V. Bulaev and D. Loss, Phys. Rev. Lett. 95, 076805 (2005). 